\title{
Soil properties affecting weed distribution in spring cereal and vegetable fields
}

\author{
Raimo Erviö, Seppo Hyvärinen, Leila-Rittta Erviö and Jukka Salonen \\ Erviō, R. ${ }^{1}$, Hyvărinen, S. ${ }^{1}$, Erviö, L.-R. ${ }^{2}$ \& Salonen, J. ${ }^{2}$ 1994. Soil properties \\ affecting weed distribution in spring cereal and vegetable fields. Agricultural \\ Science in Finland 3: 497-504. (Agricultural Research Centre of Finland, ' Insti- \\ tute of Soils and Environment, and ${ }^{2}$ Institute of Plant Protection, FIN-31600 Jokioi- \\ nen, Finland.)
}

\begin{abstract}
The incidence of weed species in 482 cereal and 224 vegetable field plots in southern and central Finland was investigated. The occurrence of the 16 most common weed species was related to soil properties. Chenopodium album L., Lamium spp. L. and Fallopia convolvulus (L.) Löve were more abundant in clay than in coarse mineral or organic soils. Elymus repens (L.) Gould, Erysimum cheiranthoides L., Lapsana communis L., Myosotis arvensis (L.) Hill and Poa annua L. thrived better in coarse than in clay soils. Polygonum lapathifolium L. and Rumex spp. L. were more abundant in organic than in mineral soils, and Lamium spp. was not found at all in organic soils.

Rumex spp., Poa annua and Polygonum lapathifolium had higher densities at the lowest $\mathrm{pH}$ level, < 5.2. Lamium spp. and Myosotis spp. thrived at the highest $\mathrm{pH}$ levels. Poa annua and Spergula arvensis were most common in soils where the extractable calcium concentration was below $1000 \mathrm{mg} \mathrm{l}^{-1}$ soil.
\end{abstract}

Key words: Finnish fields, soil type, soil pH, soil organic matter content, soil extractable calcium

\section{Introduction}

A study was carried out in 1982 - 1984 to determine the occurrence of weeds in Finnish spring cereals (ERviö and SALONEN 1987) and vegetable fields. Of special interest was the importance of farming management and field factors on weed infestation. As part of this study, the present paper discusses the influence of soil types and soil properties on the occurrence of weed species in cereal and vegetable fields of southern and central Finland.

Diverse conclusions have been drawn about the effect of soil factors on the incidence of weed species. The role of soil $\mathrm{pH}$ in the abundance of some species has been reported by some workers (Ferdinandsen 1918, de VRIES 1934, TrautmanN 1954). Low $\mathrm{pH}$ may be a restrictive factor in the success of many pretentious weed species, some of which are considered to be calciphiles.

The importance of available nutrients for weed abundance has also been investigated. It would seem however, that physical soil properties such as porosity, aeration, waterholding capacity and humus content have a more marked effect on the abundance of weeds than do chemical soil properties (EllenberG 1951, ReHDER 1959). 
Table 1. Distribution of soil types in different localities.

\begin{tabular}{|c|c|c|c|c|c|c|}
\hline \multirow[b]{3}{*}{ Localities } & \multicolumn{6}{|c|}{ Number of plots in soil-type groups } \\
\hline & \multicolumn{3}{|c|}{ Cereal fields } & \multicolumn{3}{|c|}{ Vegetable fields } \\
\hline & $\begin{array}{c}\text { Coarse } \\
\text { mineral } \\
\text { soils }\end{array}$ & $\begin{array}{l}\text { Clay } \\
\text { soils }\end{array}$ & $\begin{array}{c}\text { Organic } \\
\text { soils }\end{array}$ & $\begin{array}{c}\text { Coarse } \\
\text { mineral } \\
\text { soils }\end{array}$ & $\begin{array}{l}\text { Clay } \\
\text { soils }\end{array}$ & $\begin{array}{c}\text { Organic } \\
\text { soils }\end{array}$ \\
\hline Archipelago & 4 & 18 & 2 & 8 & 2 & 2 \\
\hline Southwestern Finland & 100 & 200 & 23 & 20 & 7 & 74 \\
\hline Southern Finland & 1 & 22 & - & 17 & 48 & 4 \\
\hline Eastern Finland & 38 & - & 5 & 19 & 1 & 1 \\
\hline Western Finland & 6 & 14 & 4 & 3 & 3 & 1 \\
\hline Central Finland & 20 & 3 & 2 & 6 & - & 3 \\
\hline Ostrobothnia & 12 & 1 & 7 & 4 & 1 & - \\
\hline Total & 181 & 258 & 43 & 77 & 62 & 85 \\
\hline
\end{tabular}

\section{Material and methods}

A weed survey was conducted in spring cereal and vegetable fields in ten localities in southern and central Finland in 1982-1984. A detailed description of the localities and data on spring cereals were given in previous publications (ERVIÖ and SALONEN 1987, SALONEN and ERVIÖ 1988, SALONEN 1993). In those papers the occurrence of weeds was analysed from 3-5 plots in every field but here weed numbers have been used only for the plots from which the soil samples were taken. The material has been divided into seven locality groups (Table 1). Weed data on vegetable fields were gathered in the same localities as the spring cereal data. The spring cereals were wheat, oats and barley and the most common vegetable crops were carrot, onion, cabbage and swede.

The number of weeds was counted from a total of 482 cereal plots in 265 fields and from 224 vegetable plots in 112 fields on 379 farms (Table 1). The weed assessment was made on $0.25 \mathrm{~m}^{2}$ circular sample plots during the second half of July. The interdependences between weed densities and soil properties were studied. Only the 16 most common weed species were analysed.

Soil samples were taken from the plough layer
$(0-20 \mathrm{~cm})$ by mixing five separate auger pricks together. They were dried and ground to pass through a 2-mm sieve. Soil $\mathrm{pH}\left(\mathrm{H}_{2} \mathrm{O}\right)$, organic matter content and extractable calcium, potassium, magnesium and phosphorus concentrations were measured on all soil samples. In addition, the particle-size analysis was made on 101 samples to confirm that the soil type was correct.

Soil $\mathrm{pH}\left(\mathrm{H}_{2} \mathrm{O}\right)$ was determined on a soil:water $\mathrm{V} / \mathrm{V}$ suspension of $1: 2.5$. The organic carbon content of the soil was determined by the automated dry ashing method. The samples were combusted in an oxygen atmosphere and the $\mathrm{CO}_{2}$ gas formed was measured with a solid-state infrared detector. A conversion factor of 1.73 was used to convert organic carbon to organic matter (O.M.).

Calcium and other macronutrients were extracted from the soil using a $0.5 \mathrm{~N}$ ammonium acetate $0.5 \mathrm{~N}$ acetic acid (AAAc) solution ( $\mathrm{pH} 4.65$ ), the extraction ratio being 1:10 V/V (VUORINEN and MÄKITIE 1955). Concentrations of $\mathrm{Ca}, \mathrm{K}, \mathrm{Mg}$ and $\mathrm{P}$ were measured on the soil extracts employing an inductive coupled plasma emission spectrometer (ICP). The concentrations are given as milligrams per litre of soil.

The occurrence of weeds was compared in the soil-type groups. The material was divided into three soil groups: coarse mineral, clay and organic soils. The first group comprised sand, fine- 
sand, silt and glacial till soils, and the last one peat, mould and gyttja soils. A soil sample was classified as peat if the O.M. content was at least $40 \%$, as mould if the O.M. content was $20-40 \%$ and as gyttja if O.M. was at least $6 \%$ in lake sediment soil. The soil types were distributed unequally between localities. Thus, in some localities of southern Finland there were no organic soil plots and in eastern Finland no clay soil plots (Table 1).

Differences in the occurrence of weeds in the various soil type groups were analysed with Tukey's HSD multiple range test $(P=0.05)$. The statistically significant differences in the tables are based on the analysis with log-transformed data. The results given in the tables are backtransformed from the logarithmic mean values. Data transformation was used to achieve normal distribution and homogeneity of variances.

\section{Results and discussion}

\section{Soil type}

The analysis of weed densities (plants $\mathrm{m}^{-2}$ ) indicated differences in the occurrence of weed species in the various soil types better than did the analysis of weed biomass. It apparently takes only a single big weed plant to raise the biomass ranges to an unreasonably high level. In a previous paper (SALONEN 1993) examining the total weed density, not the density by species, differences were found between soil types in the total weed biomass but not in the total weed density.

Here, divergent densities of weed species were found in different soil-type groups (Table 2). However, the densities of some weeds differed in the soils of cereal and vegetable fields, indicating the strong influence of crop type on weed incidence (cf. ANDREASEN et al. 1991). The abundance of weeds varied from one species to another. Chenopodium album had the highest density of all species, averaging 74 plants $\mathrm{m}^{-2}$ in the clay soils of vegetable fields. The densities of seven weed species averaged only 1 plant $\mathrm{m}^{-2}$ or less.

Chenopodium album and Lamium spp. in both cereal and vegetable fields and Fallopia convolvulus in cereal fields were more dense in clay soils than in coarse mineral or organic soils. Similar results have been reported for Lamium (REHDER 1959, ANDREASEN et al. 1991), and Fallopia convolvulus (GRANSTRÖM 1962). In contrast, Korsmo (1925) reported that Chenopodium album and Lamium purpureum occured in equal densities in all kinds of soil, and REHDER (1959) considered Fallopia convolvulus an indifferent species.

Weed species that thrived better in coarse mineral than clay soils were Elymus repens, Lapsana communis, Myosotis arvensis and Poa annua and, in addition, Erysimum cheiranthoides and Spergula arvensis in cereal fields. ANDREASEN and STREIBIG (1990) and ANDREASEN et al. (1991) likewise found a negative correlation between clay content and the density of Elymus repens, Myosotis arvensis and Spergula arvensis. KORSMO (1925) and, later, REHDER (1959) and GRANSTRÖM (1962) showed that Spergula arvensis was particularly prevalent in light soil types. Elymus repens, Myosotis arvensis and Spergula arvensis were characterized for mineral soil species by RAATIKAINEN and RAATIKAINEN (1983); this was not found in the present data.

Chenopodium album and Galium spp. were more abundant in mineral than in organic soils, especially in vegetable fields. Lapsana communis and Viola arvensis occurred more abundantly in coarse mineral than in organic soils, and Lamium spp. was not found at all in organic soils. In the whole material, Polygonum lapathifolium was more common in organic than in mineral soils as was Rumex spp. in cereal fields. GRANSTRÖM (1962) and RAATIKAINEN and RAATIKAINEN (1983) also reported a greater abundance of $P$. lapathifolium in organic than in mineral soils, and Streibig et al. (1984) found a positive correlation between soil organic matter and Rumex acetosella. Mukula et al. (1969) found 30 plants $\mathrm{m}^{-2}$ of $P$. lapathifolium in organic soils but only 6 and 12 plants $\mathrm{m}^{-2}$ in clay and coarse mineral soils, respectively. In this study the density of Spergula arvensis was higher in organic soils than in clay soils. 
Table 2. Weed density in different soil types. Arithmetic means and geometric means, with superscripts denoting statistically significant $(\mathrm{P}<0.05)$ differences.

\begin{tabular}{|c|c|c|c|c|c|c|}
\hline & \multicolumn{6}{|c|}{ Plants $\mathrm{m}^{-2}$} \\
\hline & \multicolumn{3}{|c|}{ Cereal fields } & \multicolumn{3}{|c|}{ Vegetable fields } \\
\hline & $\begin{array}{c}\text { Coarse } \\
\text { mineral } \\
\text { soils }\end{array}$ & $\begin{array}{l}\text { Clay } \\
\text { soils }\end{array}$ & $\begin{array}{l}\text { Organic } \\
\text { soils }\end{array}$ & $\begin{array}{c}\text { Coarse } \\
\text { mineral } \\
\text { soils }\end{array}$ & $\begin{array}{l}\text { Clay } \\
\text { soils }\end{array}$ & $\begin{array}{l}\text { Org. } \\
\text { soils }\end{array}$ \\
\hline Chenopodium album $\mathrm{L} .{ }^{\prime}$ & $\begin{array}{c}18 \\
2.3^{b^{2}}\end{array}$ & $\begin{array}{c}26 \\
6.2^{\mathrm{a}}\end{array}$ & $\begin{array}{c}8 \\
2.1^{\mathrm{b}}\end{array}$ & $\begin{array}{c}56 \\
8.4^{a}\end{array}$ & $\begin{array}{c}74 \\
4.8^{a}\end{array}$ & $\begin{array}{c}14 \\
1.6^{\mathrm{b}}\end{array}$ \\
\hline Elymus repens (L.) Gould & $\begin{array}{c}18 \\
1.6^{\mathrm{a}}\end{array}$ & $\begin{array}{c}2 \\
0.5^{b}\end{array}$ & $\begin{array}{c}8 \\
1.2^{\mathrm{a}}\end{array}$ & $\begin{array}{c}23 \\
2.5^{a}\end{array}$ & $\begin{array}{c}7 \\
0.8^{\mathrm{b}}\end{array}$ & $\begin{array}{c}6 \\
0.7^{\mathrm{b}}\end{array}$ \\
\hline Erysium cheiranthoides $\mathrm{L}$. & $\begin{array}{c}16 \\
1.1^{\mathrm{a}}\end{array}$ & $\begin{array}{c}4 \\
0.7^{b}\end{array}$ & $\begin{array}{c}9 \\
0.9^{\text {ab }}\end{array}$ & $\begin{array}{c}9 \\
1.7^{\mathrm{a}}\end{array}$ & $\begin{array}{c}3 \\
1.0^{\mathrm{ab}}\end{array}$ & $\begin{array}{c}2 \\
0.9^{\mathrm{b}}\end{array}$ \\
\hline Fallopia convolvulus (L.) Löve & $\begin{array}{c}3 \\
0.9^{b}\end{array}$ & $\begin{array}{c}7 \\
1.7^{\mathrm{a}}\end{array}$ & $\begin{array}{c}2 \\
0.8^{\mathrm{b}}\end{array}$ & $\begin{array}{c}4 \\
0.6^{b}\end{array}$ & $\begin{array}{c}1 \\
0.5^{b}\end{array}$ & $\begin{array}{r}3 \\
1.5^{a}\end{array}$ \\
\hline Galeopsis spp. L. & $\begin{array}{c}8 \\
2.0^{b}\end{array}$ & $\begin{array}{c}17 \\
3.6^{\mathrm{a}}\end{array}$ & $\begin{array}{c}25 \\
3.4^{\mathrm{a}}\end{array}$ & $\begin{array}{c}5 \\
1.0^{\mathrm{b}}\end{array}$ & $\begin{array}{c}4 \\
0.9^{b}\end{array}$ & $\begin{array}{c}12 \\
2.0^{3}\end{array}$ \\
\hline Galium spp. L. & $\begin{array}{c}3 \\
0.5^{b}\end{array}$ & $\begin{array}{c}8 \\
0.8^{a}\end{array}$ & $\begin{array}{c}0 \\
0.6^{\mathrm{ab}}\end{array}$ & $\begin{array}{c}6 \\
0.8^{a}\end{array}$ & $\begin{array}{c}3 \\
0.7^{a}\end{array}$ & $\begin{array}{l}<1 \\
0.4^{\text {b }}\end{array}$ \\
\hline Lamium spp. L. & $\begin{array}{c}3 \\
0.6^{b}\end{array}$ & $\begin{array}{c}8 \\
0.8^{a}\end{array}$ & $\begin{array}{c}0 \\
0.4^{\mathrm{b}}\end{array}$ & $\begin{array}{c}2 \\
0.5^{b}\end{array}$ & $\begin{array}{c}6 \\
0.9^{a}\end{array}$ & $\begin{array}{c}0 \\
0.4^{a}\end{array}$ \\
\hline Lapsana communis $\mathrm{L}$. & $\begin{array}{c}16 \\
1.7^{\mathrm{a}}\end{array}$ & $\begin{array}{c}4 \\
0.9^{b}\end{array}$ & $\begin{array}{c}9 \\
0.5^{b}\end{array}$ & $\begin{array}{c}14 \\
1.2^{a}\end{array}$ & $\begin{array}{c}<1 \\
0.4^{\mathrm{b}}\end{array}$ & $\begin{array}{l}<1 \\
0.5^{b}\end{array}$ \\
\hline Myosotis arvensis (L.) Hill & $\begin{array}{c}8 \\
1.2^{\mathrm{a}}\end{array}$ & $\begin{array}{c}2 \\
0.6^{\mathrm{b}}\end{array}$ & $\begin{array}{c}1 \\
0.6^{b}\end{array}$ & $\begin{array}{c}2 \\
0.6^{\mathrm{a}}\end{array}$ & $\begin{array}{l}<1 \\
0.5^{a b}\end{array}$ & $\begin{array}{l}<1 \\
0.4^{\text {b }}\end{array}$ \\
\hline Poa aпnua $\mathrm{L}$. & $\begin{array}{c}5 \\
0.6^{\mathrm{a}}\end{array}$ & $\begin{array}{c}1 \\
0.4^{b}\end{array}$ & $\begin{array}{c}1 \\
0.5^{a b}\end{array}$ & $\begin{array}{l}29 \\
1.7^{\mathrm{a}}\end{array}$ & $\begin{array}{c}1 \\
0.4^{b}\end{array}$ & $\begin{array}{r}9 \\
1.1^{\mathrm{a}}\end{array}$ \\
\hline Polygonum lapathifolium L. & $\begin{array}{c}1 \\
0.5^{b}\end{array}$ & $\begin{array}{c}1 \\
0.5^{b}\end{array}$ & $\begin{array}{c}9 \\
0.9^{\mathrm{a}}\end{array}$ & $\begin{array}{c}2 \\
0.5^{b}\end{array}$ & $\begin{array}{c}1 \\
0.5^{b}\end{array}$ & $\begin{array}{c}7 \\
1.4^{a}\end{array}$ \\
\hline Rumex spp. L. & $\begin{array}{c}1 \\
0.5^{\mathrm{b}}\end{array}$ & $\begin{array}{c}<1 \\
0.4^{\mathrm{b}}\end{array}$ & $\begin{array}{c}7 \\
0.7^{\mathrm{a}}\end{array}$ & $\begin{array}{l}<1 \\
0.5^{a}\end{array}$ & $\begin{array}{c}0 \\
0.4^{a}\end{array}$ & $\begin{array}{c}1 \\
0.5^{a}\end{array}$ \\
\hline Sonchus spp. L. & $\begin{array}{c}2 \\
0.6^{\mathrm{a}}\end{array}$ & $\begin{array}{c}1 \\
0.6^{\mathrm{ab}}\end{array}$ & $\begin{array}{c}0 \\
0.4^{\mathrm{b}}\end{array}$ & $\begin{array}{c}8 \\
1.0^{\mathrm{a}}\end{array}$ & $\begin{array}{c}2 \\
0.6^{\mathrm{ab}}\end{array}$ & $\begin{array}{c}1 \\
0.5^{b}\end{array}$ \\
\hline Spergula arvensis $\mathrm{L}$. & $\begin{array}{c}15 \\
1.1^{\mathrm{a}}\end{array}$ & $\begin{array}{c}2 \\
0.6^{b}\end{array}$ & $\begin{array}{c}5 \\
1.1^{\mathrm{a}}\end{array}$ & $\begin{array}{c}2 \\
0.8^{a b}\end{array}$ & $\begin{array}{c}1 \\
0.6^{b}\end{array}$ & $\begin{array}{c}6 \\
1.2^{a}\end{array}$ \\
\hline Stellaria media (L.) Vill. & $\begin{array}{c}30 \\
4.0^{\mathrm{ab}}\end{array}$ & $\begin{array}{c}27 \\
5.8^{\mathrm{a}}\end{array}$ & $\begin{array}{c}9 \\
2.0^{b}\end{array}$ & $\begin{array}{c}17 \\
2.2^{\mathrm{b}}\end{array}$ & $\begin{array}{c}14 \\
3.7^{\mathrm{b}}\end{array}$ & $\begin{array}{c}30 \\
8.6^{a}\end{array}$ \\
\hline Viola arvensis $\mathrm{L}$. & $\begin{array}{c}17 \\
2.6^{\mathrm{a}}\end{array}$ & $\begin{array}{c}12 \\
2.2^{\mathrm{ab}}\end{array}$ & $\begin{array}{c}9 \\
1.2^{\mathrm{b}}\end{array}$ & $\begin{array}{c}21 \\
3.2^{\mathrm{a}}\end{array}$ & $\begin{array}{c}18 \\
1.9^{\text {ab }}\end{array}$ & $\begin{array}{c}6 \\
1.0^{b}\end{array}$ \\
\hline
\end{tabular}

\footnotetext{
${ }^{1}$ Including Atriplex patula $\mathrm{L}$.

${ }^{2}$ Densities marked with different superscripts differ from each other significantly $(\mathrm{P}<0.05)$ between the soil classes in cereal or vegetable fields.
}

\section{Soil pH}

The soils were divided into six classes by $\mathrm{pH}$ level. The incidence of some weed species was affected by soil $\mathrm{pH}$ (Tables 3 and 5). The nega- tive significances of the dependence ( $F$ value) of weed density on soil $\mathrm{pH}$ were highest for Polygonum lapathifolium, Rumex spp. and Spergula arvensis (Table 5). The abundance of Rumex species was most clearly affected by the soil $\mathrm{pH}$. 
Table 3. Week density in different soil pH classes in cereal and vegetable fields. Arithmetic means and geometric means, with superscripts denoting statistically significant $(\mathrm{P}<0.05)$ differences between classes.

\begin{tabular}{|c|c|c|c|c|c|c|}
\hline \multirow[b]{2}{*}{ Weed } & \multicolumn{6}{|c|}{$\begin{array}{c}\text { Plants m }{ }^{-2} \\
\text { Soil } \mathrm{pH}\left(\mathrm{H}_{2} \mathrm{O}\right)\end{array}$} \\
\hline & $\begin{array}{c}<5.2 \\
\mathrm{n}=79\end{array}$ & $\begin{array}{l}5.2-5.4 \\
\mathrm{n}=100\end{array}$ & $\begin{array}{l}5.5-5.7 \\
\mathrm{n}=137 \\
\end{array}$ & $\begin{array}{l}5.8-6.0 \\
\mathrm{n}=161\end{array}$ & $\begin{array}{l}6.1-6.4 \\
\mathrm{n}=115\end{array}$ & $\begin{array}{c}>6.4 \\
\mathrm{n}=114\end{array}$ \\
\hline Chenopodium album & $\begin{array}{l}22.7 \\
12.6^{\mathrm{a}^{1}}\end{array}$ & $\begin{array}{c}58.5 \\
4.6^{\mathrm{b}}\end{array}$ & $\begin{array}{c}25.1 \\
4.2^{\mathrm{b}}\end{array}$ & $\begin{array}{c}18.8 \\
4.0^{\mathrm{b}}\end{array}$ & $\begin{array}{c}26.3 \\
5.1^{\mathrm{b}}\end{array}$ & $\begin{array}{c}30.4 \\
4.6^{b}\end{array}$ \\
\hline Lamium spp. & $\begin{array}{l}0.5 \\
0.4^{b}\end{array}$ & $\begin{array}{l}0.7 \\
0.5^{b}\end{array}$ & $\begin{array}{l}2.3 \\
0.6^{\mathrm{ab}}\end{array}$ & $\begin{array}{l}4.2 \\
0.7^{\mathrm{ab}}\end{array}$ & $\begin{array}{l}6.7 \\
0.7^{\mathrm{ab}}\end{array}$ & $\begin{array}{c}10.1 \\
0.9^{\mathrm{ab}}\end{array}$ \\
\hline Lapsana communis & $\begin{array}{l}4.8 \\
0.5^{\mathrm{c}}\end{array}$ & $\begin{array}{l}4.8 \\
0.6^{b c}\end{array}$ & $\begin{array}{l}5.3 \\
1.0^{\mathrm{ab}}\end{array}$ & $\begin{array}{l}10.5 \\
1.1^{\mathrm{ab}}\end{array}$ & $\begin{array}{l}9.4 \\
1.2^{\mathrm{a}}\end{array}$ & $\begin{array}{l}8.7 \\
1.0^{\mathrm{ab}}\end{array}$ \\
\hline Myosotis arvensis & $\begin{array}{l}0.4^{\mathrm{b}} \\
0.4^{\mathrm{b}}\end{array}$ & $\begin{array}{l}1.1 \\
0.5^{\mathrm{b}}\end{array}$ & $\begin{array}{l}5.0 \\
0.7^{\mathrm{ab}}\end{array}$ & $\begin{array}{l}4.1 \\
0.8^{a}\end{array}$ & $\begin{array}{l}4.3 \\
1.0^{\mathrm{a}}\end{array}$ & $\begin{array}{l}1.8 \\
0.7^{a c}\end{array}$ \\
\hline Poа аппиа & $\begin{array}{c}20.5 \\
1.2^{\mathrm{a}}\end{array}$ & $\begin{array}{l}6.8 \\
0.8^{\mathrm{ab}}\end{array}$ & $\begin{array}{l}6.2 \\
0.6^{b}\end{array}$ & $\begin{array}{l}0.4 \\
0.4^{\mathrm{c}}\end{array}$ & $\begin{array}{l}3.7^{b} \\
0.5^{b}\end{array}$ & $\begin{array}{l}5.4 \\
0.6^{\mathrm{b}}\end{array}$ \\
\hline Polygonum lapathifolium & $\begin{array}{l}9.0 \\
1.2^{\mathrm{a}}\end{array}$ & $\begin{array}{l}2.8 \\
0.7^{\mathrm{b}}\end{array}$ & $\begin{array}{l}1.4 \\
0.6^{\mathrm{b}}\end{array}$ & $\begin{array}{l}0.8 \\
0.5^{\mathrm{b}}\end{array}$ & $\begin{array}{l}0.8 \\
0.5^{\mathrm{b}}\end{array}$ & $\begin{array}{l}1.5 \\
0.5^{\mathrm{b}}\end{array}$ \\
\hline Rumex spp. & $\begin{array}{l}5.4 \\
0.7^{\mathrm{a}}\end{array}$ & $\begin{array}{l}0.4^{\mathrm{b}} \\
0.4^{\mathrm{b}}\end{array}$ & $\begin{array}{l}0.2 \\
0.4^{\mathrm{b}}\end{array}$ & $\begin{array}{l}0.2 \\
0.4^{b}\end{array}$ & $\begin{array}{l}0.1 \\
0.4^{b}\end{array}$ & $\begin{array}{l}0.1 \\
0.4^{b}\end{array}$ \\
\hline Spergula arvensis & $\begin{array}{l}6.2 \\
1.2^{\mathrm{a}}\end{array}$ & $\begin{array}{l}3.2 \\
0.9^{\text {ab }}\end{array}$ & $\begin{array}{c}16.6 \\
1.0^{\mathrm{a}}\end{array}$ & $\begin{array}{l}2.6 \\
0.7^{\mathrm{ab}}\end{array}$ & $\begin{array}{l}2.4 \\
0.6^{b}\end{array}$ & $\begin{array}{l}3.2 \\
0.6^{\mathrm{b}}\end{array}$ \\
\hline
\end{tabular}

${ }^{\prime}$ Densities marked with different superscripts differ from each other significantly $(\mathrm{P}<0.05)$ between pH classes.

Their density was highest at $\mathrm{pH}$ values below 5.2 , being nearly ten times as high as at $\mathrm{pH}$ values at least 5.2. Rumex acetosella has also been mentioned as an indicator of soil acidity (NIELSEN 1926, Ellenberg 1950, RehDER 1959 and Streibig et al. 1984). This opinion is not held by all authors, however (KIVINEN 1931 and ÅsLANDER 1941). Another species abundant in acid soil was Polygonum lapathifolium, as also mentioned by FERDINANDSEN (1918) and REHDER (1959); ELLENBERG (1950) in contrast considered it a pHindifferent species. The density of Poa annua was noticeably more abundant at $\mathrm{pH}<5.2$ than at $>5.5$. Spergula arvensis weeds were numerous at pH $5.5-5.7$ but significantly less so at $\mathrm{pH}>6.1$.

Species thriving at high $\mathrm{pH}$ levels were $\mathrm{La}$ mium spp., Lapsana communis and Myosotis spp.
Lamium species grew most abundantly at $\mathrm{pH}>6.4$. Nielsen (1926), Ellenberg (1950), REHDER (1959) and ANDREASEN et al. (1991) also reported Lamium spp. thriving at high soil $\mathrm{pH}$ levels. The density of Myosotis spp. was higher at $\mathrm{pH}>5.8$ class than at $\mathrm{pH}<5.5$. However, there was no clear indication of its demand for high pH. ElLENBERG (1950) mentioned Myosotis arvensis as an indifferent species.

\section{Extractable nutrients}

There was no distinct, consistent relationship between extractable potassium and phosphorus and weed density. Soil-extractable calcium seemed to affect the densities of some weed species (Table 4). Elymus repens, Poa annua, Rumex spp. and Spergula arvensis grew at their highest density 
Table 4. Weed density in different extractable calcium classes in cereal and vegetable fields. Arithmetic means, and geometric means, with superscripts denoting statistically significant $(\mathrm{P}<0.05)$ differences.

\begin{tabular}{|c|c|c|c|c|c|}
\hline \multirow[t]{3}{*}{ Weed } & \multicolumn{5}{|c|}{ Plants $\mathrm{m}^{-2}$} \\
\hline & \multicolumn{5}{|c|}{ Extractable Ca concentration $\mathrm{mg} \mathrm{l}^{-1}$ soil } \\
\hline & $\begin{array}{l}<1000 \\
n=73\end{array}$ & $\begin{array}{c}1000-1499 \\
n=162\end{array}$ & $\begin{array}{c}1500-1999 \\
\mathrm{n}=181\end{array}$ & $\begin{array}{c}2000-2499 \\
\mathrm{n}=112\end{array}$ & $\begin{array}{l}\geq 2500 \\
\mathrm{n}=178\end{array}$ \\
\hline Elymus repens & $\begin{array}{l}18.4 \\
1.5^{\mathrm{a}^{\mathrm{a}}}\end{array}$ & $\begin{array}{l}10.5 \\
1.1^{\mathrm{ab}}\end{array}$ & $\begin{array}{l}8.7 \\
0.9^{\mathrm{ab}}\end{array}$ & $\begin{array}{l}9.8 \\
0.9^{\mathrm{ab}}\end{array}$ & $\begin{array}{l}6.1 \\
0.8^{\mathrm{b}}\end{array}$ \\
\hline Myosotis arvensis & $\begin{array}{l}1.2 \\
0.8^{\mathrm{ab}}\end{array}$ & $\begin{array}{l}1.2 \\
1.0^{\mathrm{a}}\end{array}$ & $\begin{array}{l}0.6 \\
0.7^{\text {ab }}\end{array}$ & $\begin{array}{l}0.5 \\
0.7^{\text {ab }}\end{array}$ & $\begin{array}{l}0.2 \\
0.5^{\mathrm{b}}\end{array}$ \\
\hline Lapsana communis & $\begin{array}{c}13.8 \\
1.2^{\mathrm{a}}\end{array}$ & $\begin{array}{l}13.6 \\
1.4^{a}\end{array}$ & $\begin{array}{l}5.9 \\
0.8^{\mathrm{bc}}\end{array}$ & $\begin{array}{l}3.3 \\
0.8^{\mathrm{bc}}\end{array}$ & $\begin{array}{l}3.9 \\
0.6^{c}\end{array}$ \\
\hline Poa annua & $\begin{array}{r}28.9 \\
1.3^{\mathrm{a}}\end{array}$ & $\begin{array}{l}1.1 \\
0.5^{\mathrm{b}}\end{array}$ & $\begin{array}{l}5.3 \\
0.6^{\mathrm{b}}\end{array}$ & $\begin{array}{l}5.5 \\
0.6^{\mathrm{b}}\end{array}$ & $\begin{array}{l}2.0 \\
0.5^{b}\end{array}$ \\
\hline Spergula arvensis & $\begin{array}{c}28.7 \\
1.4^{a}\end{array}$ & $\begin{array}{l}4.3 \\
1.0^{\mathrm{cc}}\end{array}$ & $\begin{array}{l}4.9 \\
0.8^{b c}\end{array}$ & $\begin{array}{l}1.1 \\
0.6^{b}\end{array}$ & $\begin{array}{l}1.9 \\
0.7^{\mathrm{bc}}\end{array}$ \\
\hline Stellaria media & $\begin{array}{c}15.8 \\
2.0^{\mathrm{b}}\end{array}$ & $\begin{array}{l}27.1 \\
3.9^{\text {ab }}\end{array}$ & $\begin{array}{r}23.0 \\
3.2^{\mathrm{a}}\end{array}$ & $\begin{array}{r}31.6 \\
2.8^{\mathrm{a}}\end{array}$ & $\begin{array}{c}24.5 \\
3.1^{\mathrm{a}}\end{array}$ \\
\hline
\end{tabular}

' Densities marked with different superscripts differ from each other significantly $(\mathrm{P}<0.05)$ between Ca classes.

in the lowest $\mathrm{Ca}$ class $\left(<1000 \mathrm{mg} \mathrm{l}^{-1}\right.$ soil $)$. The abundance of Poa annua differed most clearly. Negative significances of the dependence ( $F$ value) of weed density on the concentration of extractable calcium of soil were found only in Elymus repens, Lapsana communis and Myosotis arvensis (Table 5). According to de VRIES (1934), however, Spergula arvensis did not indicate a low calcium concentration of soil as it grew well in soil rich in calcium, too. Stellaria media was the only species to occur in a significantly higher density in the highest Ca classes (over $1500 \mathrm{mg}$ ) than in the class below $1000 \mathrm{mg} \mathrm{Ca}$.

In conclusion, the occurrence of several weed species differed between soil types. Crop type influenced the incidence of all weeds, and soil $\mathrm{pH}$ and the extractable calcium concentration the incidence of some. The density of Poа апnиа, Polygonum lapathifolium and Rumex spp. was highest in the lowest $\mathrm{pH}$ class but that of Lamium spp. in the highest one. The occurrence of some species, e.g. Poa annua and Spergula arvensis, can apparently be reduced with liming as they had a very high density in the lowest extractable calcium class.

Table 5. Statistical significance of dependence ( $\mathrm{F}$ value, ANOVA), of soil pH and concentration of extractable calcium of soil on weed density. $+=$ ascending, $-=$ descending.

\begin{tabular}{lcc}
\hline & \multicolumn{2}{c}{ F values } \\
\cline { 2 - 3 } Weed & $\begin{array}{c}\text { Soil } \\
\mathrm{pH}\end{array}$ & $\begin{array}{c}\text { Extractable } \\
\text { calcium }\end{array}$ \\
\hline Chenopodium album & $15.3^{* * *_{+}}$ & $10.2^{* *+}$ \\
Elymus repens & - & $8.1^{* *}+$ \\
Lamium spp. & $18.7^{* * *+}$ & - \\
Lapsana communis & $15.1^{* * *+}$ & $21.0^{* * *+}$ \\
$\begin{array}{l}\text { Myosotis arvensis } \\
\text { Poa annua }\end{array}$ & $14.5^{* * *+}$ & $19.2^{* * *}+$ \\
Polygonum lapathifolium & $17.9^{* * *+}$ & - \\
Rumex spp. & $28.2^{* * *+}$ & - \\
Stellaria media & $21.4^{* * *+}$ & - \\
\hline$* *=\mathrm{P}<0.01$ & $5.4^{* *+}$ & $6.6^{* *}+$ \\
\hline
\end{tabular}




\section{References}

Andreasen, C. \& Streibig, J.C. 1990. Impact of soil factors on weeds in Danish cereal crops. Proceeding EWRS Symposium, Integrated Weed Management in Cereals. Helsinki. p. 53-59.

-, Streibig, J.C. \& HaAs, H. 1991. Soil properties affecting the weed distribution of 37 weed species in Danish fields. Weed Research 31: 181-187.

ElLenBERG, H. 1950. Unkrautgemeinschaften als Zeiger für Klima und Boden. Landwirtschaftliche Pflanzensoziologie Band I. 141 p.

- 1951. Landwirtschaftliche Standortkartierung auf pflanzengemässer Grundlage. Zeitschrift für Pflanzenernährung Düngung Bodenkunde 53: 204-224.

Erviö, L.-R. \& SAlonen, J. 1987. Changes in the weed population of spring cereals in Finland. Annales Agriculturae Fenniae 26: 201-226.

FERDINANDSEN, C. 1918. Undersögelser over danske ukrudsformationer paa mineraljorder. Tidsskrift for Planteavl. 25: 629-919.

GRANSTRŌM, B. 1962. Studier över ogräs i vårsådda grödor. Summary: Studies on weeds in spring crops. Statens Jordbruksförsök. Meddelande Nr 130. 188 p.

KIVINEN, E. 1931. Ahosuolaheinän (Rumex acetosella) kasvupaikkojen reaktiosta. Referat: Über die Reaktion der Standorte des Kleinen Ampfers (Rumex acetosella). Journal of the Scientific Agricultural Society of Finland 3: 10-16.

Korsmo, E. 1925. Ugress i nutidens jordbruk. Oslo. 635 p.

Mukula, J., RaATIKainen, M., LALlukKa, R. \& RAATIKAINEN, T. 1969. Composition of weed flora in spring cereals in Finland. Annales Agriculturae Fenniae 8: $59-110$.

NIELSEN, N.C. 1926. Ukrudsvegetationen som Vejledning ved Undersogelser over Mineraljordens Kalktrang. Beretning N.J.F.s Kongres, Oslo. 12 p.

RAATIKAINEN, M. \& RAATIKAINEN, T. 1983. Syysviljan viljelystä ja sen vaikutuksesta rikkaruohoihin Suomes- sa. Abstract: Survey on cultivation of winter wheat and its effect on weeds in Finland. Journal of the Scientific Agricultural Society of Finland. 55: 384-423.

REHDER, H. 1959. Über die Beziehungen der Ackerunkräuter zur Bodenart sowie zum Säuregrad, Phosphorsäure- und Kaligehalt des Bodens im Raum um Hamburg. Abhandlungen und der Verhandlungen des Naturwissenschaftlichen Vereins in Hamburg. Bd.III: 55-85.

SALONEN, J. 1993. Weed infestation and factors affecting weed incidence in spring cereals in Finland - a multivariate approach. Agricultural Science in Finland 2: 525-536.

- \& ERviö, L.-R. 1988. Efficacy of chemical weed control in spring cereals in Finland. Weed Research 28: 231-235.

Streibig, J.C., Gottschau, A., Dennis, B., HaAs, H. \& MolgaARD, P. 1984. Soil properties affecting weed distribution. Proceedings of 7th International Symposium on Weed Biology, Ecology and Systematics. Paris. p. 147-154.

TrautmanN, W. 1954. Über den Einfluss von Bodenreaktion, Kali und Phosphorsäure auf die Verteilung der Ackerkräuter im Göttinger Gebiet. Zeitschrift für Pflanzenernährung Düngung Bodenkunde 66: 247-261.

VRIES de, O. 1934. Unkräuter und Säuregrad. Zeitschrift für Pflanzenernährung, Düngung und Bodenkunde 13: 356-360.

Vuorinen, J. \& MÄKITIE, O. 1955. The method of soil testing in use in Finland. Agrogeological Publications No 63: 1-44.

ÅsLANDER, A. 1941. Rödsyran som markindikator. Summary: Sheep sorrel as an indicator plant. Svensk Botanisk Tidskrift 35: 219-238.

Manuscript received February 1994 


\title{
SELOSTUS
}

\section{Maaperäominaisuuksien vaikutus rikkakasvilajien esiintymiseen}

\author{
Raimo Erviö, Seppo Hyvärinen, Leila-RiItTa Erviö ja JukKa Salonen
}

\author{
Maatalouden tutkimuskeskus
}

Rikkakasvilajien esiintymisselvityksessä vuosina 19821984 otettiin tutkituilta näytealoilta myös maanäytteet, joiden maalajin ja kemiallisten ominaisuuksien merkitystä rikkakasvilajistoon ja yksilöiden määrään selvitettiin. Yhteensä 379 tilalta kerättiin 482 näytettä kevätvilja- ja 224 näytettä vihanneskasvilohkoilta.

Jauhosavikka ja peipit esiintyivät savimailla runsaampina kuin karkeilla kivennäismailla tai eloperäisillä mailla. Sen sijaan karkeita kivennäismaita suosivia lajeja olivat juolavehnä, ukonnauris, linnunkaali ja nurmikka. Ukontatar ja suolaheinä esiintyivät eloperäisillä mailla runsaam- pina kuin kivennäismailla, mutta peippejä ei löytynyt eloperäisiltä mailta lainkaan.

Suolaheinien ja ukontattaren lukumäärät olivat suurimmat alimmassa $\mathrm{pH}$-luokassa $(\mathrm{pH}<5,2)$. Peipit ja orvokit menestyivät parhaiten korkealla $\mathrm{pH}$-tasolla. Kylänurmikka oli selvästi runsaampi alle $1000 \mathrm{mg}$ helppoliukoista kalsiumia sisältäneessä kuin vähintään $1000 \mathrm{mg}$ kalsiumia sisältäneissä luokissa, kun sen sijaan pihatähtimön esiintyminen oli juuri vastakkainen. Maan helppoliukoisen kaliumin, magnesiumin tai fosforin määrä ei vaikuttanut rikkakasvilajien esiintymiseen. 